\title{
Home-Based Risk of Falling Assessment Test Using a Closed-Loop Balance Model
}

\author{
Johannes C. Ayena, Helmi Zaibi, Martin J.-D. Otis, and Bob-Antoine J. Ménélas
}

\begin{abstract}
The aim of this study is to improve and facilitate the methods used to assess risk of falling at home among older people through the computation of a risk of falling in real time in daily activities. In order to increase a real time computation of the risk of falling, a closed-loop balance model is proposed and compared with One-Leg Standing Test (OLST). This balance model allows studying the postural response of a person having an unpredictable perturbation. Twenty-nine volunteers participated in this study for evaluating the effectiveness of the proposed system which includes seventeen elder participants: ten healthy elderly (68.4 $\pm \mathbf{5 . 5}$ years), seven Parkinson's disease (PD) subjects $(66.28 \pm 8.9$ years $)$, and twelve healthy young adults (28.27 \pm 3.74 years). Our work suggests that there is a relationship between OLST score and the risk of falling based on center of pressure measurement with four low cost force sensors located inside an instrumented insole, which could be predicted using our suggested closed-loop balance model. For long term monitoring at home, this system could be included in a medical electronic record and could be useful as a diagnostic aid tool.
\end{abstract}

Index Terms-Biomechanisms, elderly, falls, one-leg standing test, Parkinson's disease, tether-release test.

\section{INTRODUCTION}

$\mathbf{F}$ ALLS are a major concern in the field of public health because of their impact on the physical and physiological state of the person. Indeed, falls represent the third leading cause of chronic disability worldwide [1] and cause about $81 \%-98 \%$ of hip fractures [2]. In 2009 , their total cost was between $0.85 \%$ and $1.5 \%$ of the total health spending in the United States, Australia and the European Union [2]. In addition to physical damage and their high cost, falls leave a psychological impact because of the fear of falling again and a lack of self-confidence to keep the balance during unexpected situations. As a result, recurrent fallers need training with an

Manuscript received August 19, 2015; revised November 18, 2015; accepted December 03, 2015. Date of publication December 17, 2015; date of current version nulldate. This work was supported in part by the Natural Sciences and Engineering Research Council of Canada (NSERC), under Grant 418235-2012.

J. C. Ayena and H. Zaibi are with the Department of Applied Sciences, University of Quebec at Chicoutimi, Chicoutimi, QC, G7H 2B1 Canada (e-mail: cossoun-johannes.ayena1@uqac.ca; helmi.zaibi1@uqac.ca).

M. J.-D. Otis is with the Department of Applied Sciences, University of Quebec at Chicoutimi, QC, G7H2B1, Canada and also with REPARTI Centre of Laval University, Ville de Québec, QC, G1V 0A6 Canada (e-mail: martin otis@uqac.ca).

B.-A. J. Ménélas is with the Department of Computer Sciences and Mathematics, University of Quebec at Chicoutimi, Chicoutimi, QC, G7H 2B1, Canada (e-mail: bob-antoine-jerry_menelas@uqac.ca).

Color versions of one or more of the figures in this paper are available online at http://ieeexplore.iee.org.

Digital Object Identifier 10.1109/TNSRE.2015.2508960 automatic evaluating system at home, as suggested in this paper.

The identification of individuals who are at risk of falling due to balance issues becomes a major challenge in falls prevention. The risk of falling among elderlies or persons with neurodegenerative disorder such as Parkinson's and Ataxia disease is generally assessed by clinical tests such as Timed Up and Go test [3], One-Leg Standing test [4], Tinetti test [5] or Berg Balance Scale [6]. Most of these tests are performed in a clinical environment; this could be costly for both time and money for the patient, its family or for the health care system. Falls consequences are even intensified by the demographic change. In a near future, there should be more geriatric patients and probably fewer professionals will be available to assess the risk of falling in a clinical environment. As a result, recently, some studies have attempted to bring the risk of falling assessment at home [7], [8]. These previous research works indicated a home-based evaluation has a good potential since it could allow identifying an unstable balance and gait at an early stage. However, the experimental protocol and the devices used are not often straightforward. In addition, clinical values of gait abnormalities identified can not be interpreted by a layman. Moreover, although many aspects of balance and gait can influence risk of falling, a critical factor is the ability of participant to respond effectively at balance perturbations. These perturbations can arise from external disturbance (such as wind and slope) or the type of ground (such as sand and snow) on which the participant performs the tests. For example, soft surfaces can represent an important risk of falling factor [9], [10], and they are not taken into account in the clinical tests assessment at home. Since we evaluate the impact of environmental disturbances, the instrumented One-Leg Standing Test (OLST) is the most appropriate for home-based assessment tools compared to Timed Up and Go, or Tinetti tests. Berg Balance Scale (BBS) test demonstrated its inefficiency in the determination of people who did fall and people who did not fall [11] and OLST is the most convenient for home testing [12], [13]. Indeed, the test of the standing position allows the study of human capacity to maintain postural balance. This test is characterized by the center of mass (COM) displacements and deviation of the center of pressure (COP) in the base of support [14], [15]. Moreover, the ability to maintain balance could include a swing motion of the body at the level of the ankle. Thus, there is a quasi-static motion similar to a controlled inverted pendulum system.

In this context, the research focuses on the identification of various parameters to take into account in an automated risk of fall evaluation. To achieve this goal, many of models were designed to study the effect of sensory inputs and disturbances of 
the body sway [16]-[18]. Overall, they include a posture control representation which encompasses proprioceptive, visual and vestibular mathematical model in Laplace domain. In addition, these research interests to study the external disturbances such as obstacles on the floor, the movement of a ship or bus and internal disturbances such as breathing, heart rate, muscle tremors, dizziness or vertigo. In doing that, different methods are performed to simulate these disturbances to describe the postural response and the compensatory balance reactions in an uncontrolled environment.

In this paper, in order to increase the robustness of the risk of fall evaluation in real-time, we propose in the first part, a new closed-loop balance model which represents the human body at standing position. The tether-release system is used as an external Heaviside input in order to identify the intrinsic parameters of the model. This model will be used to understand the mechanism underlying the risk of falling and to find a score related to the risk computed in real time. Moreover, this model is used to simulate the balance capability for home monitoring and diagnostic aid. In the second part, we propose an automatic version of One-Leg Standing Test. This last is executed on different types of ground as perturbations. Since we need a home-based system, we implemented this test on a Smartphone and used an instrumented insole with Bluetooth capability. The types of ground such as concrete, parquet, sand and gravel are used to be similar to an everyday environment. Therefore, our test enhances the ability to differentiate the risk of falling related to balance issues and external perturbations. The main contribution of our work is to evaluate the relevance of a balance model included in an inexpensive home-based system to compute a risk of falling and, to train and to monitor an elderly with balance issue. The second contribution is to characterize the parameters of balance recovery under soil perturbations.

Following a review of the state of the art, we described the primary contribution of this paper, a new risk of falling assessment method based on our instrumented insole, an home-based Android serious game on which the risk of falling is computed by a new closed-loop balance model. Such a system could be used for long term home monitoring using a medical electronic record. The first evaluation shows encouraging results which are then discussed for usage at home.

\section{RELATED WORK}

First, a brief overview of instrumented tests using in both gait and balance disorders assessment is presented. In this, we cover mobile evaluation systems such as instrumented insoles. Thereafter, clinical tests used in this study are detailed where we address the clinical evaluation which could be used for home monitoring.

\section{A. Technologies for Gait and Balance Disorder Analysis}

In the last decade, instrumented tests for gait analysis have been widely used to assess risk of falling by evaluating gait parameter's variation. Several types of shoes equipped with instrumented insoles were developed using various technologies [19], [20]. Instrumented insole demonstrates the capabilities to compute walking parameters such as pressure located at the heel and toes, and the midstance time [21]. Other studies present an instrumented insole able to detect gait phases such as swing time, double support time, stride length and cadence [22]. The instrumented gait analysis system enables planning and assessment of risk of falling among older people and is considered as a useful tool for quantifying locomotors performance in people with Parkinson's or Ataxia Disease [23], [24]. Zampieri et al. [8] studied body-worn sensors for mobility testing at home versus a laboratory testing situation. Their results show that home testing is feasible. Recently, a Smartphone software has proved to be an effective tool for showing clinical tests parameters at home [25]. Other studies showed that a Smartphone-based system may be used for assessing risk of falling [12] and for training users at maintaining balance [26] over different types of ground [27] by using a serious game. These recent studies have shown that mobile systems can be used for assessing clinical test parameters. However, most of these recent research works usually compares clinical values for differentiating the groups in their works without a formal evaluation with elder participants.

Other studies have been directed toward the development of new instrumented insoles for clinical tests with low-cost and often with wireless communications such as presented in [28], [29]. Those insoles were used for ergonomic evaluation in standing posture and for lower limb prosthetic. Previous studies did not consider the environment of the participant. It is known that the type of ground can affect the gait [10]. Moreover, some studies relate the effects of unstable surfaces such as rocks [30] on the gait parameters. The type of ground could become a significant factor that should be taken into account in fall risk assessment. Our main contribution is for using such instrumented insole for a home-based system designed in order to assess risk of falling in standing position tests. Then, calibrate a personal closed-loop balance model in order to evaluate the risk of falling in daily activities. The identified parameter values of the balance model represent some indices related to the progression of balance disorder. Therefore, our system needs a clinical test that could be translated in a serious game on a Smartphone. Those tests are discussed in the next section.

\section{B. Standing Position Tests}

Several studies showed that dynamic balance has become important for understanding the risk of falling level in clinical settings. This section emphasis One-Leg standing Test (OLST) and focuses on other standing position tests relevant to be included in our falls risk assessment system.

1) One-Leg Standing Test: Many clinical studies showed that the One-Leg Standing Test (OLST) is a single task performance and can be used to determine balance issues such as neurological disease, muscular weakness, sensory-motor deficits among elderly [4], [31]. This traditional test also named unipodal test measures the time in seconds (related to a score), which is considered as a level of balance stability. Participants unable to perform this test for at least five (5) seconds are at increased risk of falling. The researchers stated that a time greater than twenty (30) seconds shows a very low risk of falls [31]. However, using only the time in this test is not quite as discriminating for medical decisions, such as adapting the drug dose, prescribe a different drug or adapting the training level. It becomes necessary 
to measure additional parameters and give a score, to the clinician, related to the risk of fall based on an accurate simulation of the balance ability using a model. Indeed during the test, the ability of participant to maintain the strength and weight evenly distributed on the foot is essential for balance assessment. The literature has shown that older people who present an unstable balance have a greater center of pressure (COP) sway [32]. COP is defined as the point location of the vertical ground reaction, and is often used to identify a balance deficit [33]. The COP displacements in standing posture become a parameter which could be taken into account in risk of falling assessment. Several studies have used a variety of COP measures to predict a risk of falling [34], [35]. They associate postural control capability with balance which is used to describe the body's ability to adjust the center of pressure near the center of mass. The center of pressure has been commonly used as cues of postural stability in standing position. To our best knowledge, no work has attempted to evaluate risk of falling by computing a score over different types of ground with the clinical OLST by using a Smartphone-based system comprising an instrumented insole. Moreover, there is no study related to the understanding of balance mechanism associated to OLST able to give an accurate simulation of the risk of fall.

2) Other Standing Position Tests Used for Balance Model Evaluation: The control of postural stability during standing is similar to that of the control of an inverted pendulum. In fact, Peterka [14] uses control theory with a classic law (in a form of a proportional-integral-derivative (PID) compensator) to address the balance model. The author shows that both balance and neuromuscular system answer varies depending on the proportional $\left(K_{p}\right)$ and derivative $\left(K_{d}\right)$ gains of the compensator, and it is weakly influenced by integral gain $\left(K_{I}\right)$. This is explained by the influence of these gains on a closed-loop system (control and stability theory).

a) An increase of the proportional gain (usually associated to a gain margin) moves the root locus toward its corresponding zero in a complex Laplace's plane. The proportional gain is bounded by the Routh-Hurwitz stability criterion. This gain then could be associated to the balance transient response after a Heaviside perturbation.

b) The derivative gain is associated to the damping ratio, which change both the natural frequency and the overshoot of the system. This gain could then be associated to the human body sway. As this gain decrease, the body sway increase in frequency and amplitude.

c) The integral term could be associated to the capability to track a zero degree in order to stay in a right posture. Meaning that this gain has a small influence on body sway: the result depends on how this parameter is identified. However, its impact is more related to the steady state response than the transient response.

The model of Peterka [14] represents a fixed position on a rigid and uniform surface and considers both sensory and neuromuscular system as a simple PID compensator. This may be because of this consideration that the sensory system is not given in the model it offers. To study a more complete model, van der Kooij et al. [36] proposed another model that serves to study the effect of sensory disturbance on the swing of human. Despite the use of sensory disturbance in their system, the controller was able to stabilize the model of an unstable standing human with neural time delays of $100 \mathrm{~ms}$. The authors concluded that their model was capable of studying and quantifying multi-sensory integration in human stance control. Ersal and Sienko [16] have developed a mathematical model to describe the human balance without focusing on the different sensory systems while Mahboobin et al. [37] developed another model based on a mechanism of a visual, proprioceptive and vestibular reweighting. The latter model helps to give explanation underlying the way that the person learns to react against the disturbances caused by a moving surface. In these models, external disturbances are driven by rotation [17], [18] or a horizontal displacement of a platform under the feet [16], [38] or a combination of both [36], [18]. In the same context, Mohapatra et al. [39] studied another type of disturbance. Their method consists of attaching a load to an aluminum pendulum. This pendulum is fixed at its upper end to the ceiling and is located in front of the participant. Subsequently, it is released leading to a disturbance at the participant's shoulders. This experiment was used to study the role of visual perception [39], the altered proprioception [40] and the impact of different surfaces [41] in the generation of anticipatory and compensatory corrections during the maintenance of posture. In fact, this experience affirmed that both foam and wobble board induce body instability and diminish the somatosensory input, as compared to rigid surface. These results confirm the work of Patel et al. [42]. This has proved that the variability of torque in the lateral direction is greater on a foam surface than on a solid surface. Thereby, they showed that standing on foam is considered as a multi-directional balance perturbation. This result confirms the usage of different type of soil for evaluating our system. Others research drew on a different type of cable release. Their method is to attach a cable to the human body that supports it against the gravitational force. At an unpredictable moment, this cable is released to react against the risk of falling created by this type of action [43]-[45]. This experience has shown the effect of arm swing in regaining balance with a single step under intense perturbation [45]. Moreover, it allows the study of the influence of novel safety flooring systems on the compensatory balance reactions of older people [44]. However, in their study, the soil types do not reflect a real environment. Indeed, they do not represent intense disturbances that can occur to a person when performing daily activities. Our main objective in this part was to determine the anticipated postural adjustments to restore the position of the body after an unpredictable disturbance.

\section{PRoposed SYSTEM}

This section presents a Smartphone-based system used for balance training and for long term monitoring. As a closed-loop balance model is used in this system, a system identification method for each user is required. The identification methodology uses tether-release system which could be executed in a clinic environment. Once the parameters identified, this model should increase the robustness for the computation of a risk of falling. In the following, we present our instrumented insole and then the others apparatus able to compute a risk of falling. 


\section{A. Instrumented Insole}

This apparatus is an intelligent system, recently developed, labelled ACHILE. ACHILE stands for Active Human-Computer Interface for Locomotion Enhancement. A schematic of the instrumented insole prototype used is shown in [12], [46], [47]. It aims at preventing accidental falls related to conditions of the physical environment (slippery ground, steep slope, etc.), or abnormalities of its gait. This device counts a set of sensors such as accelerometers (located in electronic board), force sensors and bending variable sensor. The sensors located inside this insole were selected in order to create an instrumented system capable of sensing many parameters that characterize both gait and balance parameters. All these sensors are exploited to compute a risk level associated to a risk of falling. A 16-bits architecture microcontroller is included for computing, in real time, the risk level using different algorithms such as neural network [48], fuzzy logic [49] and our closed-loop balance model presented in this paper.

To assess force distribution under foot, Force Sensitive Resistors (FSRs) were placed over the insole at strategic pressure position. Two FSRs were placed at the heel, one at right $\left(\mathrm{F}_{h r}\right)$ and the second at left $\left(\mathrm{F}_{h l}\right)$. Two others FSRs were placed at the toes, one at left $\left(\mathrm{F}_{t l}\right)$ and the second at right $\left(\mathrm{F}_{t r}\right)$ [47]. In sum, this system measures the forces applied at four points under the foot. The force sensors data variation recorded by the smartphone during the OLST has been used in order to assess the participant's stability.

\section{B. Home Monitoring Using a Serious Game Based on OLST}

A user-friendly interface was designed using the OLST procedure. The main software is running as a serious game for training balance. The risk level computed by our algorithm is displayed as a score to be improved at each usage. The evolution of the score is stored for further analysis. Some strategy could be used to increase the ludic (playfulness) such as a tai chi context and imitation of Flamingo. OLST software records raw data signals from four FSR sensors in order to estimate user performance that is doing the test. The software incorporates two main sections which are: 1) instructions on how to properly complete this clinical test, 2) data analysis and results visualization. As the user is standing balanced on one leg, the test could begin by pressing start button. A countdown timer starts from this event, which is the normative time [31] for the user to perform the OLST. Our software stops the test and data are recorded at the end of the normative time required. The daily usage of OLST software allows a remote monitoring of elderly and also could inform about the impact of drugs or rehabilitation interventions on people with Parkinson, Ataxia and other related balance disorder disease. The login and password of user are registered into data-base in order to showing its progression by comparing performance history and current score with the highest ones performed previously or in last days. The user can make countless tries knowing data are always recorded. For user safety, especially older people, and also for smooth running of the test, another person should activate OLST software.

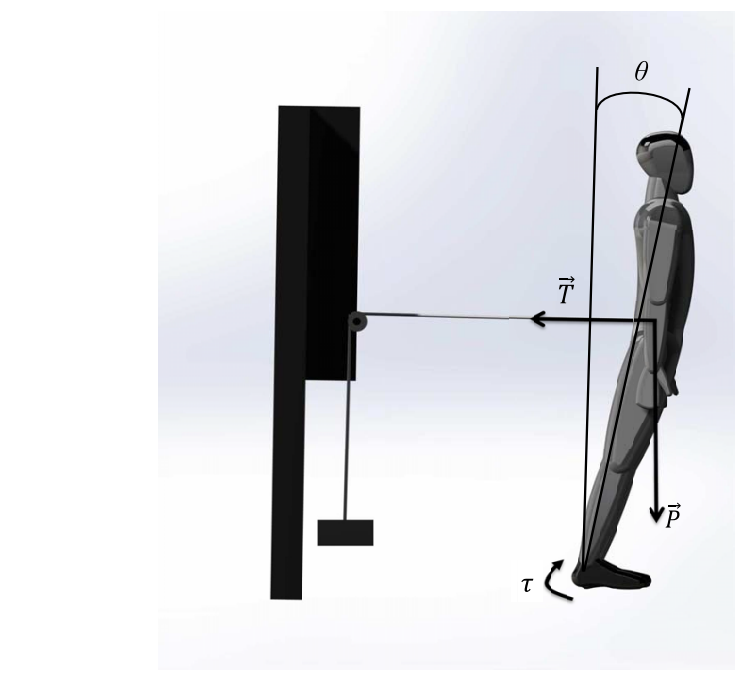

Fig. 1. Tether-release system used for the second standing test.

TABLE I

Characteristics OF PARTICIPANTS IN THIS STUdY

\begin{tabular}{|c|c|c|c|}
\cline { 2 - 4 } \multicolumn{1}{c|}{} & $\begin{array}{c}\text { Healthy Elderly } \\
(\mathrm{n}=10)\end{array}$ & Young $(\mathrm{n}=12)$ & $\begin{array}{c}\text { PD subjects } \\
(\mathrm{n}=7)\end{array}$ \\
\hline Age (years) & $68.4 \pm 5.50$ & $28.27 \pm 3.74$ & $66.28 \pm 8.90$ \\
\hline Gender $(\mathrm{M} / \mathrm{F})$ & $4 \mathrm{M} / 6 \mathrm{~F}$ & $10 \mathrm{M} / 2 \mathrm{~F}$ & $3 \mathrm{M} / 4 \mathrm{~F}$ \\
\hline Height $(\mathrm{cm})$ & $170.1 \pm 16.49$ & $174.8 \pm 9.62$ & $166.7 \pm 20.12$ \\
\hline Weight $(\mathrm{Kg})$ & $73.49 \pm 6.20$ & $70.16 \pm 9.94$ & $76.78 \pm 7.08$ \\
\hline
\end{tabular}

\section{Closed-Loop Balance Model Identification With a Tether-Release System}

The human balance should be modeled in order to compute a risk of fall. The apparatus, shown in Fig. 1, is mainly composed of a pulley around which a rope is wound and a load equal to five percent (5\%) of subject weight as suggested in [39]-[41]. This methodology will give the model parameters of the human balance.

\section{ExPERIMENTAL Methodology}

To study postural stability under quasi-static conditions, postural sway was assessed for each participant. The two following steps explain the experimental methodology used in both tests.

1) Firstly, we asked one of the authors of this study (a healthy young participant) to remain in standing position on the rigid ground and wear a belt attached approximately at the tenth thoracic vertebra. This belt is attached to a cable which is wound around a pulley and connected to a load (about $5 \%$ of the subject's body weight). In addition, a Smartphone is attached to the back of his thigh in order to measure the angle $\theta$. Then, we asked him to make an angle $\theta$ relative to the vertical line (show Fig. 1). In an unpredictable moment, the cable is released (the tensile force is then null). At the same time, Smartphone software records the angle variation of the body. Twelve trials of this process have been performed by the same person in order to identify our model.

2) Secondly, 29 volunteers, which includes 17 elderly (10 healthy, seven PD subjects) and 12 healthy young adults, have performed the OLST (show characteristics in Table I). 
Each PD subject was diagnosed by a movement disorders neurologist at state 2 or 3 . Healthy elderly (subjects without Parkinson's disease) were physically independent (University staff and other people outside University) while the healthy young adults were students. The evaluation was carried out in our laboratory. Criteria for inclusion were as follows: 1) elderly subjects were between 59 and 79 years old while 2) young adults were between 22 and 35 years old.

All participants involved in this study were informed about the experimental protocol and gave written consent before particpating. The experience and consent form had been previously approved by the local ethics committee (certificate number 602 . 434.01). The OLST procedure was performed as follows.

a) In a first time, the test was performed at normative time with young participants over four types of ground such as concrete, gravel, sand, and parquet. Two types of ground (concrete and parquet) are encountered at domestic environment and the others were chosen according to its difficulty to maintain balance.

b) Since older people have difficulty to maintaining their balance, OLST was only performed at normative time with them over concrete. Elder participants were asked to maintain balance at least five seconds up to the normative time. Each participant in this study was asked to put the instrumented insole in their right shoe and to do the test using OLST software two times. It was instructed to stand upright on the right foot without support of the upper extremities by looking straight ahead. The OLST was performed with eyes open condition in all cases above. Overall, the number of seconds a participant was able to maintain a stable position was recorded by the software. The test ended (pressed stop button) when one of these conditions are reached: 1) the foot up touches the support leg, 2) the foot up touches the ground, or 3) the arms touch something for improving balance control.

c) After the OLST, the participants have also walked three (3) meters over each type of ground in order to make a diagnostic by the clinician.

\section{IDENTIFICATION OF THE Closed-LooP HuMAN BALANCE MODEL}

In this section, we present first the closed-loop human balance model. This model is identified with the pivoting angle $\theta$ of participants after the perturbation is removed (the tensile force in the cable is released). This section explains how the model is designed and then identified.

The human body standing on a type of ground can be approximated with the classical model of an inverted pendulum (show Fig. 1). The equation of motion of the system is given by:

$$
J \theta^{\prime \prime}=\tau+m g L \sin \theta-T L \cos \theta
$$

where $\theta$ " is the second derivative of the pivoting angle $(\theta)$ of human body, $J$ : is the moment of inertia of a body at ankle joint, $T:$ is the tensile force in the cable, $g$ : is the gravity acceleration, $m$ : is the mass of participant, $\tau$ : is the torque produced at the ankle joint, and $L$ : is the distance between ankle joint and center of mass of body.

We suppose that: $-0.07<\theta<0.24$ (radian), then we get

$$
\begin{aligned}
\cos \theta & =1-\left(\frac{\theta^{2}}{4}\right)-\left(\frac{\theta^{4}}{24}\right)-\left(\frac{\theta^{6}}{720}\right)-\cdots \cdots \\
& \rightarrow \cos \theta=1 ; \text { error }_{\cos }<3 \% \\
\sin \theta & =\theta-\left(\frac{\theta^{3}}{6}\right)-\left(\frac{\theta^{5}}{120}\right)-\left(\frac{\theta^{7}}{5040}\right)-\cdots \cdots \\
& \rightarrow \sin \theta=\theta ; \text { error }_{\sin }<3 \%
\end{aligned}
$$

By using the result (2) and (3) in (1), then (1) is simplified as

$$
\begin{aligned}
J \theta^{\prime \prime} & =\tau+m g L \theta-T L \\
J \theta(s) s^{2} & =\tau(s)+m g L \theta(s)-T L \\
\theta(s) & =\left[\frac{1}{\left(J s^{2}-m g L\right)}\right][\tau(s)-T L] .
\end{aligned}
$$

In this study, we hypothesis that the foot sole makes an insignificant angle with the ground during pivoting of the body. Moreover, during the rotation at the ankle while the body goes forward, we assumed that the load rises at a constant speed and therefore its acceleration is zero. On the other part, we considered that the cable is inextensible, massless and it is wound on pulley without friction. Therefore, the tensile force of the cable is equal to the weight of the load. Using superposition principle on (6), we can find this transfer function $H(s)$ as follows:

$$
H(s)=\frac{1}{\left(J s^{2}-m g L\right)} .
$$

Then, (6) will be as follows:

$$
\theta(s)=\tau H(s)-T L H(s)
$$

As indicated previously, the values of $T$ and $L$ are identified experimentally with the measurement of the ankle angle from the Tether-Release system. Then, to determine the state representation of the equation of motion of our system, it is sufficient to study the transfer function $H(s)$.

Then, using these constraints

$$
\begin{aligned}
\theta & =H(s) \tau_{\text {total }}(s) ; \tau_{\text {total }}(s)=\tau-T L \\
H(s) & =\left[\frac{\theta(s)}{\tau_{\text {total }}(s)}\right]=\frac{1}{\left(J s^{2}-m g L\right)} \\
\tau_{\text {total }} & =\theta(s)\left[J s^{2}-m g L\right]
\end{aligned}
$$

for: $\theta^{\prime}=\theta_{1}^{\prime}=\theta_{2}$ and $\theta_{2}^{\prime}=\theta_{3}$ we obtain

$$
\theta_{3}=\left[\left(\frac{1}{J}\right)(\tau-T L)\right]+\left[\left(\frac{m g L}{J}\right) \theta(t)\right] \text {. }
$$

For the simulation in MATLAB Simulink, the initial condition is located in the second integrator for the initial angle of the ankle. To design the suggested closed-loop balance model shown in Fig. 2, we used first, a transfer function representing the neuromuscular system used for an aircraft pilot [50] as follows as:

$$
H=\frac{w_{n}{ }^{2}}{\left(s^{2}+2 w_{n} \zeta+w_{n}^{2}\right)}
$$




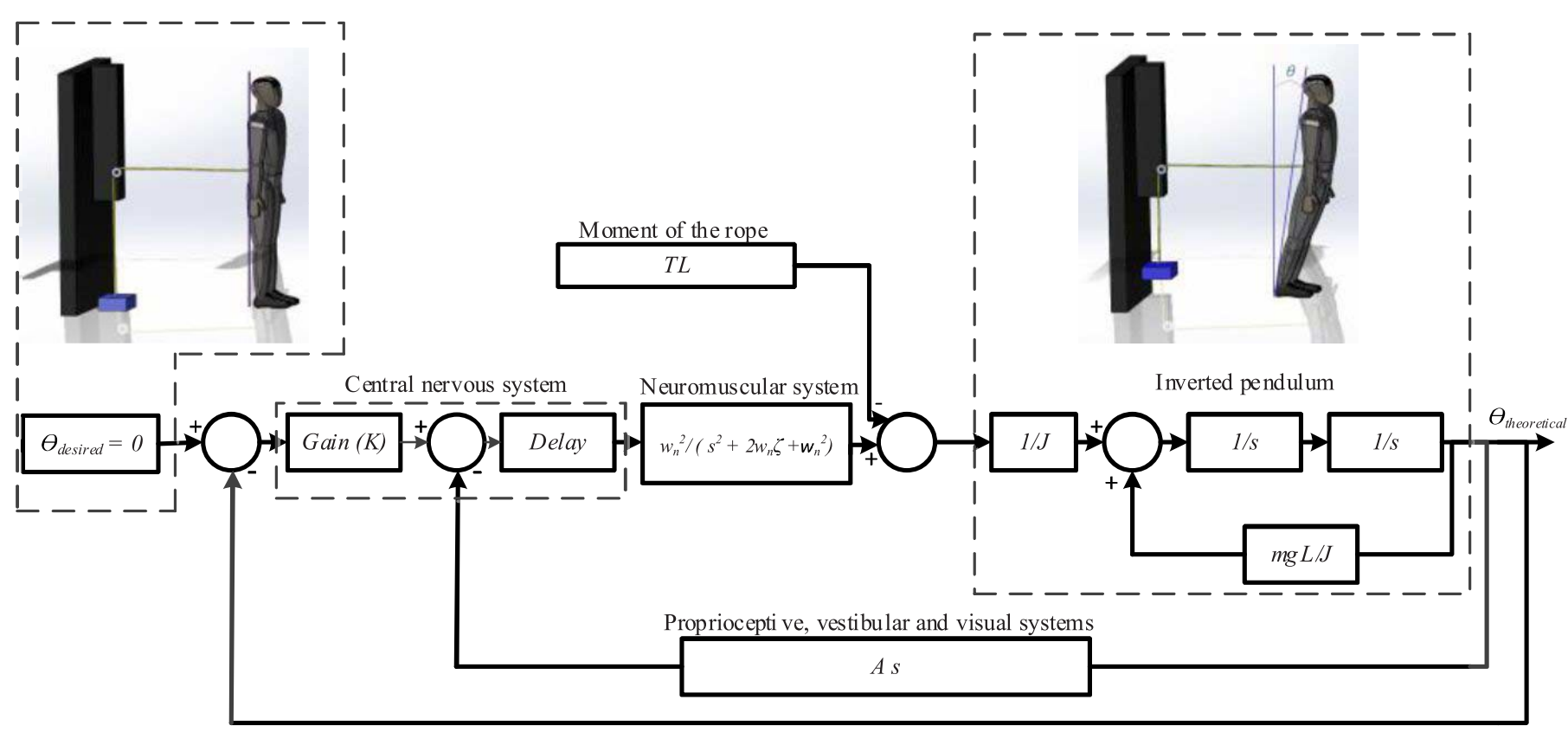

Fig. 2. Closed-loop balance Model.

where $w_{n}$ and $\zeta$ are the natural frequency and the damping ratio of neuromuscular system respectively:

Gilbert [51] has shown that an optimal damping ratio has been estimated between 0.6 and 0.7 . Then, in our study, $\zeta=0.7$ was chosen in accordance with the works presented in [52], [53]. Moreover, the widest frequency, estimated between 14 and 26 $\mathrm{rad} / \mathrm{s}[50]$, is obtained when the damping ratio is around $\zeta=0.7$. For this reason, certain types of large high-gain accelerometers are designed with added damping to achieve this value and hence, to maximize their useful frequency range [54]. However, the findings of Cenciarini et al. [55] suggest that older adults would experience a more oscillatory response to fast occurring perturbations compared to young adults. This leads us to deduce that the damping ratio is reduced among elderly. Indeed, Park et al. [56] computed the damping ratio and these findings show that this ratio is smaller among older subjects. In PD subjects, the damping ratio was $64 \%$ higher in ON-drugs $(0.18 \pm 0.05)$ than OFF-drugs $(0.11 \pm 0.04)$ [57]. The transfer function of sensory systems of our model is similar to the vestibular system of a pilot of an aircraft taken from [58]

$$
H_{\text {sensory }}=A s . .
$$

We added A (a variable without unit), since our system reacts not only by the vestibular system, but also vision and proprioception. On the other hand, to compute the delay from the transmission of nerve impulses, we consider that the average velocity of its conduction $(v)$ is equal to $49.7 \mathrm{~m} / \mathrm{s}$ [28] for a young subject. The average motor nerve impulse conduction is significantly lower among elderly $(44.2 \pm 4.1) \mathrm{m} / \mathrm{s}$ compared to young people [59]. In addition, we considered that the average value of its movement towards the spinal cord is equal to the distance between the ankle and the middle of the trunk $(\mathrm{d}=1 \mathrm{~m})$. Thus, the delay will be as follows:

$$
t_{\text {delay }}=\frac{d}{v}
$$

\section{Risk of FALling Computation}

In this section, we describe the methodology for computing a risk of falling using first the model previously described and the OLST software using an instrumented insole.

\section{A. Proposed Score Computed by the Closed-Loop Model}

This section presents the identification of the closed-loop balance model using the tether-release test (TRT) and the proposed risk of falling index computed with this model.

1) Closed-Loop Balance Model Identification: The methodology used for identifying the model was performed by varying the values of $A$ and $K$ (two variables without unit). Then, we computed the root mean square error (RMSE) of the twelve trials performed (shown Table II). The 12 trials are shown randomly in Table II and not in the order in which the tests were performed. The model is identified based on the smallest RMSE value obtained. Moreover, after this last procedure (the use of RMSE), we computed the variance of error. Error is the difference between angles of model and experience. The angles from the model are equals to the experimental angles in all trials and experience $\mathrm{N}^{\circ} 5$ is best suited with low variance. In conclusion, the model corresponding to experience $\mathrm{N}^{\circ} 5(\mathrm{~A}=300$ and $K=1100$ ) is the most appropriate because it is the most suitable to the experimental data. Some values of this case are presented in Table III.

2) Simulation: After identifying the model, we simulated it by studying the ability of a human to maintain balance during the OLST over concrete. In order to take into account the disturbance of the soil, the term $T L$ in (12) has been replaced by $m_{f r}$ which represents the moment of ground reaction. We assume that

$$
m_{f r}=F_{\mathrm{r}} l
$$


TABLE II

Parameters Used for the Model Identification, RMSE Values COMPUTED AND the VARIANCE OF ERROR (ERROR IS THE DifFERENCE BETWEEN ANGLES OF MODEL AND EXPERIENCE)

\begin{tabular}{|c|c|c|c|c|}
\hline Experiences & $\mathrm{K}$ & $\mathrm{A}$ & RMSE & Variance \\
\hline 1 & 1080 & 280 & 0.7518 & 0.4721 \\
\hline 2 & 1200 & 330 & 0.4211 & 0.1226 \\
\hline 3 & 1040 & 330 & 0.5266 & 0.2303 \\
\hline 4 & 1080 & 300 & 0.5965 & 0.7251 \\
\hline 5 & 1100 & 300 & 0.1967 & 0.0375 \\
\hline 6 & 1060 & 1700 & 0.5795 & 0.2561 \\
\hline 7 & 1080 & 350 & 0.7000 & 0.3818 \\
\hline 8 & 1120 & 330 & 0.4258 & 0.1304 \\
\hline 9 & 1160 & 340 & 1.003 & 0.6517 \\
\hline 10 & 1110 & 320 & 0.4058 & 0.1214 \\
\hline 11 & 1190 & 350 & 0.7296 & 0.3058 \\
\hline 12 & 1130 & 330 & 0.9606 & 0.7132 \\
\hline
\end{tabular}

TABLE III

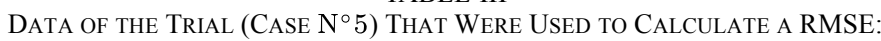
18 of 60 Values Data ( $\theta$ IN Degree) ARe Presented

\begin{tabular}{|c|c|}
\hline $\begin{array}{c}\text { Measured } \\
\text { (Experience) }\end{array}$ & $\begin{array}{c}\text { Model } \\
\text { (Theoretical) }\end{array}$ \\
\hline 3.5904 & 3.6463 \\
3.5809 & 3.6505 \\
3.5658 & 3.6675 \\
3.5701 & 3.7053 \\
3.6179 & 3.7673 \\
3.7184 & 3.8378 \\
3.8487 & 3.8912 \\
3.9806 & 3.9186 \\
4.1058 & 3.9220 \\
4.2173 & 3.9003 \\
4.2926 & 3.8405 \\
4.2953 & 3.7185 \\
4.1071 & 3.4423 \\
3.7635 & 3.1374 \\
3.4464 & 2.9165 \\
3.0586 & 2.6861 \\
2.6265 & 2.4367 \\
2.2765 & 2.1892 \\
\hline
\end{tabular}

where $F_{\mathrm{r}}$ : is the reaction force of ground and $l$ : is the distance between the heel and the point of application of $F_{\mathrm{r}}$.

It should be noted that if the human is in a standing position, the force $F_{\mathrm{r}}$ is equal to its weight. Furthermore, if its center of mass is in motion, this force will be between $80 \%$ and $120 \%$ of its body weight [60]. From this idea, we also assume that the value of $F_{\mathrm{r}}$ can be expressed as follows:

$$
F_{\mathrm{r}}=\left(\frac{B}{C}\right) m g
$$

such as

$$
B=\left(F_{h r}\right)+\left(F_{h l}\right) \text { and } C=\left(F_{t r}\right)+\left(F_{t l}\right) .
$$

From the model, we determined the angle variation of the body for each participant who performed the One-Leg Standing Test (OLST). The computation of this angle has been based on the suggested parameter $\zeta$ in the literature[55]-[57], [59], as shown in Table IV.

The weakness of muscle appears over the years. These changes among older show loss functionalities and can decrease the nerve conduction velocity (NCV). Moreover, in PD subject, basal ganglia can increase the sensory dysfunction. However,
TABLE IV

Parameters $(\zeta, w, v)$ Used for All Participants

\begin{tabular}{|c|c|c|c|}
\cline { 2 - 4 } \multicolumn{1}{c|}{} & Young & Healthy Elderly & PD subjects \\
\hline$\zeta$ & 0.7 & 0.53 & 0.18 \\
\hline$w(\mathrm{rad} / \mathrm{s})$ & 23 & 23 & 23 \\
\hline Velocity $(\mathrm{m} / \mathrm{s})$ & 49.7 & 44.2 & 44.2 \\
\hline
\end{tabular}

TABLE $\mathrm{V}$

FACTORS USED FOR COMPUTING THE MODEL SCORE RELATED TO A RISK OF FALLING

\begin{tabular}{|c|c|c|}
\hline$\sigma$ & $E_{f}$ & $C_{f}$ \\
\hline $\mathrm{X}<0.002$ & 4 & 3 \\
\hline $0.002 \leq \mathrm{X}<0.005$ & 3 & 2.5 \\
\hline $0.005 \leq \mathrm{X}<0.01$ & 2 & 2 \\
\hline $\mathrm{X} \geq 0.01$ & 1 & 1.5 \\
\hline
\end{tabular}

depending on the ON or OFF-phase of this disease, sensory and motor NCV can be normal compared to healthy elderly [61]. Given that the PD subjects involved in this study were in ON-phase during the OLST, and also sensory disturbances are not necessarily related to the OFF phase, muscular or osteo-articular conditions, we used the same value of the velocity for both groups and fixed the natural frequency at around $23 \mathrm{rad} / \mathrm{s}$.

Afterwards, we also determined the center of gravity (COG) variation, as a function of time, in order to compute a risk of falling in real time as follows:

It is known that the vertical projection (at the horizontal plane) of center of mass into the ground is often called the COG. This projection labelled $C O G_{v}$ in our study was assessed by using relation in [62]

$$
C O G_{v}=h \sin \theta
$$

where $h$ is the distance of the COM from the ankle and $\theta$ is the pivoting angle (in radians).

3) Proposed Score: To evaluate a score, we used the average of COG parameter (labelled $\mathrm{A}_{\mathrm{r}}$ ), the standard deviation $(\sigma)$ and calibration factors $\left(C_{f}\right)$. The ability to maintain stable balance labelled $E_{f}$ and the calibration factor $C_{f}$ (variables without unit) are dependent of the standard deviation and are defined in Table V.

The score was defined in the same way as the previous study in [12] where Berg Balance Scale was used:

$$
S_{\text {mod }}=\left(\frac{A_{\mathrm{r}}}{\sigma}\right)\left(E_{f} C_{f}\right)
$$

\section{B. Risk of Falling Estimation in OLST}

This section presents the COP parameters and the proposed OLST score. For better clinical assessment tests, OLST investigation focuses on exploring methods to combine the most important parameters of risk of falling into a single score. We hypothesis that unstable balance can be measured by specific COP parameters combined into this score; and could be correlated with an increased symptoms of Parkinson's disease or others balance problems among elderly.

1) COP Positions: Distributed contact forces acting on the insole surface bounded by the four sensors may be replaced by a single equivalent force $\left(F_{i}\right)$ and located at COP position $P_{i}$. 
The COP displacements on insole surface were defined by the $\mathrm{X}$ (Media-lateral) and Y (Antero-posterior) axis. The $P_{i}$ position was then calculated using relations (20) and (21) where $F_{h r} F_{h l} F_{t r} F_{t l}$ are the scalar of the four force sensors along Z-axis (perpendicular axis to insole surface); $a$ and $b$ are the distances between the force sensors along $\mathrm{X}, \mathrm{Y}$ axis respectively; and $F_{i z}$ is the total scalar force of the four sensors. All scalar force sensors are functions of time while $a$ and $b$ are constant values

$$
\begin{aligned}
P_{i x} & =\left(\frac{a}{F_{i z}}\right)\left[\left(F_{t r}+F_{h r}\right)-\left(F_{t l}+F_{h l}\right)\right] \\
P_{i y} & =\left(\frac{b}{F_{i z}}\right)\left[\left(F_{t l}+F_{t r}\right)-\left(F_{h r}+F_{h l}\right)\right] .
\end{aligned}
$$

2) Proposed OLST Score: The score was calculated following these three steps.

a) The instrumented insole allows measuring the standing time $\left(T_{s}\right)$ taken by each user. In order to assess the risk of falling by computing a score, we have compared this time with the normative time $\left(T_{N R}\right)$ which depends on age and gender. The normative values for age, gender and eyes in open or closed condition have been shown in [31].

b) $V_{P}$ represents the overall COP velocity during the test and was calculated at each data $(n)$ recorded

$$
V_{P[n]}=\left[V_{X}^{2}{ }_{[n]}+V_{Y}^{2}{ }_{[n]}\right]^{1 / 2} .
$$

The overall analysis of COP displacements is preferred compared to a separate analysis of both $X$ and $Y$-axis components [63]. The mean of COP velocity $\left(M_{V}\right)$ expressed by (22) is also calculated as follows:

$$
M_{V}=\left(\frac{1}{N}\right) \sum_{n=1}^{\mathrm{n}=N} \mathrm{~V}_{P[n]}
$$

where $\mathrm{N}$ is total data recorded during the OLST. Indeed, the mean of COP velocity is the most significant parameter that showed, in previous clinical study, a relationship between balance control and quiet standing [64]. Moreover, an inverse relationship between OLST score and the mean of COP velocity was demonstrated in [65]. The mean of COP velocity quantifies the neuromuscular activity required to maintain balance. This reflects that the postural control of subject and unstable balance in the OLST can be evaluated using a measure of mean of COP velocity in both anteroposterior and lateral directions.

c) In this study, we also computed the standard deviations of COP in each direction. The ratio $(\alpha)$ between these two values is equal to the ratio between the slope of a linear regression $\left(l_{\mathrm{r}}\right)$ and the linear correlation coefficient of Pearson $\left(l_{p}\right)$ for all COP positions on insole surface. In order to improve the assessment of real performance, and to detect a stable position or a balance issue of participant, we have multiplied the parameters in previous steps by this ratio $(\alpha)$ which can represent the ability to maintain the strength and weight even distributed on the foot. To maximise this ratio and then the result, the participant must control his balance and reduce his sway along anteroposterior and lateral directions

$$
\alpha=\left(\frac{\sigma_{y}}{\sigma_{x}}\right)=\frac{l_{\mathrm{r}}}{l_{p}}
$$

The multiplication of parameters described in these three steps provides a result ranging between zero $(0)$ and onehundred (100) corresponding inversely at different risk of falling levels. The goal was to obtain a scale according to the clinical tests scale such as Berg Balance Scale for example. The single score ( $\left.S_{\mathrm{OLS}}\right)$ could be expressed by

$$
S_{\mathrm{OLST}}=\left(\frac{T_{s}}{T_{N R}}\right)\left(\frac{1}{M_{V}}\right)\left(\frac{\sigma_{y}}{\sigma_{x}}\right)
$$

where $T_{s}$ is the time performed by the user during the OLST; $T_{N R}$ is the normative time which depends on age and gender. The normative values for age, gender and eyes in open or closed condition have been evaluated in [31]. $M_{V}$ is the mean of COP velocity; $\sigma_{y}$ and $\sigma_{x}$ are respectively the standard deviations along $\mathrm{X}$ and $\mathrm{Y}$ directions.

3) Human Perception of the Risk of Falling: After walking on the four types of ground, the participant involved in the OLST have answered simple questions related to difficult to maintain balance.

a) Of the four types of ground, on which did you had the most difficulty to walk?

b) Of the four types of ground, on which did you felt a sense of imbalance?

c) What could you say about the risk of falling level on each type of ground?

For all participants, the risk of falling level is computed as follows:

Likert scale (1-5) was awarded to the answers from each participant for each type of ground according to the risk of falling level (very low risk to very high risk). The qualitative risk level for all participants in the OLST has been computed as a mean $R_{i}$

$$
R_{i}=\left(\frac{1}{N_{J}}\right) \sum_{p=1}^{p=N_{J}} p
$$

where $N_{J}$ is the total number of participants and $p$ is the point attributed to their response.

Walking over the four types of ground (from easiest to most difficult) and the feeling of imbalance were quantified by assigning a number one over the ground where an imbalance or difficulty was noted. Then, the mean $\left(R_{i m}\right)$ was calculated for the three groups expressed as a percentage.

\section{RESULTS}

The relation between balance control and risk of falling are computed by the Smartphone software and the closed-loop balance model into a single score. The scores obtained have been analyzed by using MATLAB software. One way Analysis of Variance (ANOVA) was performed with this software in order to compare level of stability by using the dependent variable 


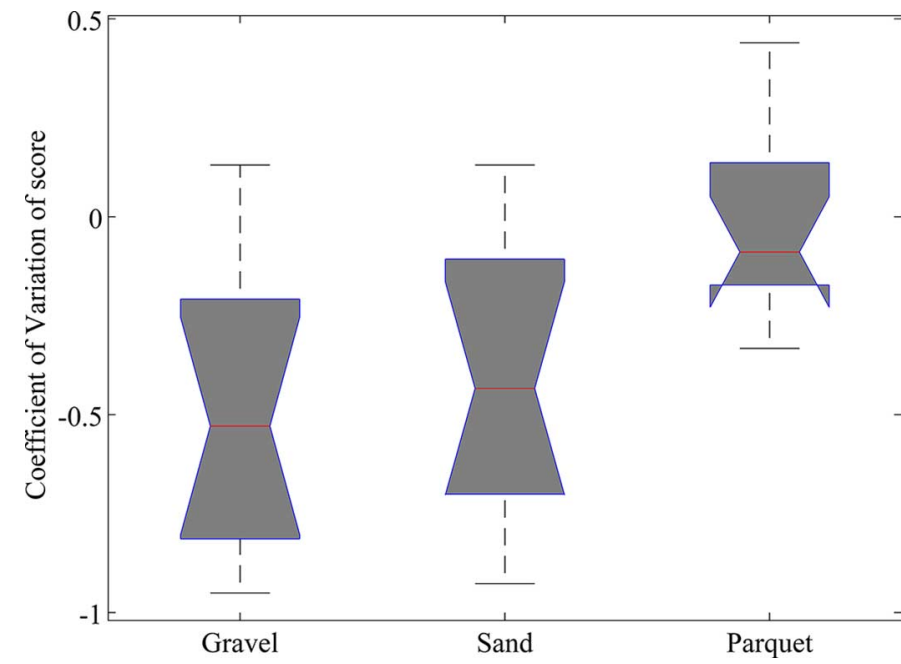

Fig. 3. Risk of falling assessment over different types of ground: the boxplot of $C V$ 's score at normative value $\mathrm{T}_{N R}$. Means are shown by the center lines, boxes indicate median and interquartile range. The $\mathrm{p}$-value was: $p=0.0025<0.05$.

(the score) and the independent variable (the type of ground). The independent variable has been divided into four groups which correspond to concrete, parquet, sand and gravel. The dependent variable could correspond inversely to three risks of falling levels (low, medium and high risk). The ANOVA was performed on an index labelled CV (Coefficient of Variation) of score which has been defined as follows:

$$
C V=\frac{\left(S_{I}-S_{b}\right)}{S_{M}}
$$

where $S_{I}$ is the score (from gravel, parquet or sand) obtained by the participant and $S_{b}$ the baseline score (score from concrete). $S_{M}$ is the OLST score average from concrete of all participants.

The ANOVA result (show Table VI) is reported as an F-statistic with its associated degrees of freedom (df) and $\mathrm{p}$-value, the mean of square (MS) and the sum of square (SS). The hypothesis is formulated about the means of the groups on that dependent variable (the $C V$ of score). The null hypothesis $H_{0}$ is that all the means of $\mathrm{CV}$ score from the three different types of ground (parquet, gravel and sand) are equals. Given that the null hypothesis is rejected if $F>F_{\text {critical }}$ at the 0.05 level of significance, the computed test statistic $F$ in our work is 7.22 , which is more than $F_{\text {critical }}=2.82(p=0.0025<0.05)$. This analysis of variance, shown in Fig. 3, leads to the conclusion that there has a significant effect related to the types of ground and risk of falling $(F(2,33)=7.22, p<0.05)$. The ANOVA analysis of CV score was performed between the three types of ground and Table VII reports their p-values. Higher severity of balance disorder was associated with lower OLST scores or lower CV's scores, which indicated more mobility deficits (as shown in Fig. 6, with black grey graph). The results from questionnaire to participants have shown that none of them in this study has proven difficult to walk on concrete or parquet. Similarly, any perceived imbalance has been notified on these two floors and gravel by young participants $(0 \%)$. Overall, the risk level was less than 1.5 (Fig. 4). $41.6 \%$ of young participants, $86 \%$ of healthy elderly and $78.56 \%$ of PD subjects have been notified to perceived imbalance and

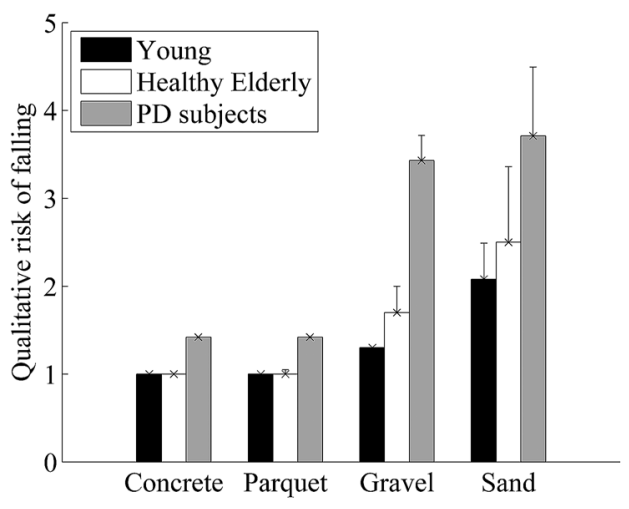

Fig. 4. Results questionnaires: 1) The average of risk of falling according to Likert scale; 2) Walk over type of ground (from easiest to most difficult) and the feeling of imbalance (this part of the results is represented as an error bar above the average bar, as noted previously: $R_{i m}$ ).

difficulty to walk over sand. $28.57 \%$ of PD subjects and $30 \%$ of healthy elderly perceived imbalance over gravel. The risk of falling remains higher over sand and gravel according to Likert scale using all participants' responses especially for elderly. As shown in Fig. 5, we determined the curves of angle variation $\left(\theta_{\text {theoretical }}\right)$. The experimental values of the angle variation are contained in an envelope formed by the two curves (min and max of all experiences). The experimental hypothesis is that the two estimates (measured and theoretical angle variation) are similar. The analysis of variances leads to this conclusion.

The raw data of each participant during the OLST have been used to simulate the balance capability with the proposed balance model. A risk of falling has been computed as shown in Fig. 6 (white bar graph).

\section{DISCUSSION}

The main purpose of this study was to improve the methods used for evaluating balance control of elderly at home especially people with Parkinson's disease. In our study, we determined the relationship between a score and a risk of falling by comparing three participants groups and evaluating the effect of the types of ground. It was observed that the type of ground did affected OLST score and therefore the risk of falling (Fig. 3). The risk of falling depends therefore on type of ground (ground properties such as compliance and coefficient of friction). By using concrete ground as baseline, the OLST scores over parquet and sand or over parquet and gravel are statistically different (Table VII). This difference is more pronounced over gravel: aggregate size of this material is bigger than sand, which has probably causes some body sway and foot motion more often. However, the risk of falling according to Likert scale is more pronounced with sand (Fig. 4) which could be due to difficulty to walk over this soil. No significant difference was found between gravel and sand ( $p=0.6126>0.05$, Table VII). It was noticed by elderlies that the balance control is different over each type of ground (Fig. 4), which means environment is an important factor in risk of falling. Most of elderly have difficulty to maintain balance until the normative value. In fact, among elderly, the left foot touch regularly the ground or support during the OLST before the normative value required except five healthy elderly and three PD subjects. These three PD 


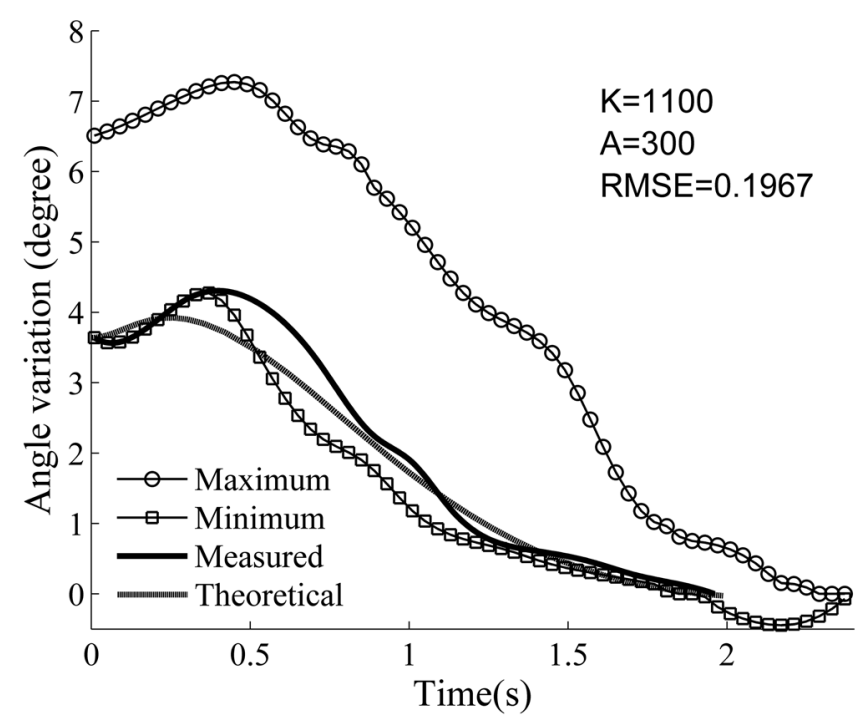

Fig. 5. Identification of the model used in the second test (TRT test): angles variation of experience $\mathrm{N}^{\circ} 5$ (measured and theoretical curves) framed by the maximum and minimum of the 12 cases.

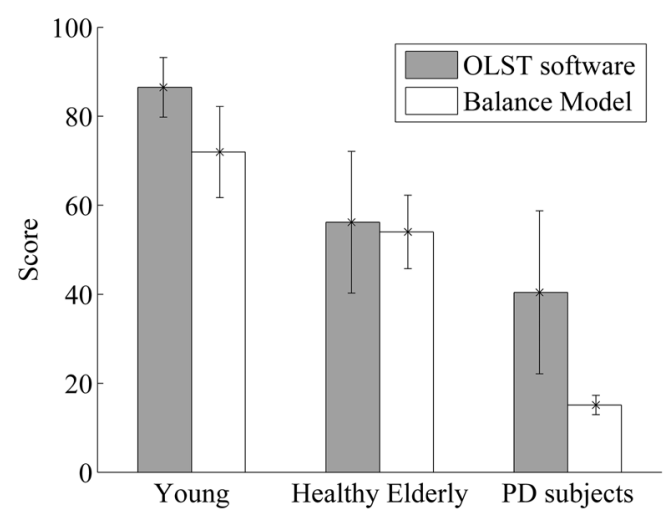

Fig. 6. Score level computed by the OLST software and the model for all participants over concrete (mean and vertical bars are standard errors).

TABLE VI

ANOVA THE OLST PERFORMED With Young PARTICIPANTS OVER THE FOUR TYPES OF GROUND

\begin{tabular}{|l|c|l|l|c|c|}
\hline \multicolumn{1}{|c|}{ Source } & SS & df & \multicolumn{1}{c|}{ MS } & F & p \\
\hline Between groups & 1.45541 & 2 & 0.72771 & 7.22 & 0.0025 \\
Within groups & 3.32633 & 33 & 0.1008 & & \\
Total & 4.78174 & 35 & & & \\
\hline
\end{tabular}

TABLE VII

p-VALUES: TYPeS OF GROUND COMPARED WiTH CONCRETE AS BASELINE IN OLST

\begin{tabular}{|c|c|c|}
\hline Gravel-Sand & Gravel-Parquet & Sand-Parquet \\
\hline 0.6126 & $0.0012^{\mathrm{a}}$ & $0.0046^{\mathrm{a}}$ \\
\hline
\end{tabular}

subjects were at state 2 or 2.5 and have a good OLST score. For enhancing the balance capability among these participants, the closed-loop balance model proposed has been also designed and simulated. Looking at Fig. 5, we observe a similarity between the variation of the angle measured during the experiments and the variation of the angle given by the model. Control subjects had greater scores for both tests compared to PD subjects in baseline condition (Fig. 6). By performing the statistical analysis on the scores, Fig. 6 also shows (at 0.05 level) no significant difference between the model and OLST software implemented (Young: $p=0.1202$, Healthy elderly: $p=0.4645$, PD subjects: $p=0.0952$ ). The main finding of this work is that this model could be used to simulate the balance capability and could be implemented inside the embedded microcontroller of the insole in real-time.

\section{CONCLUSION}

A novel methodology approach to evaluate risk of falling at home has been designed. An experiment with participants (healthy young, healthy elderly and Parkinson's disease subjects) demonstrates the feasibility of our approach. We have implemented an automatic system and proposed closed-loop balance model providing the risk of falling based on a score level. As suggested by our results, COP and COM parameters analyzed during OLST and TRT seem to be useful for evaluating risk of falling at home among elderly. The score level computed by OLST software and the model can be also used as a motivation in order to improve the physical condition of elderly. Many tests were conducted and our findings suggested that the two proposed scores could be a good candidate, which could possibly help us to evaluate and train balance among elderly and people with Parkinson's disease in the near future at home. Finally, in order to validate both scores computation related to risk of falling, we also analyzed the ground's effect on balance parameters of human. The type of ground increases the imbalance and can lead a walker to fall. The different types of ground that an elderly might walk on which must be a hint for future works to pay attention to this issue.

\section{ACKNOWLEDGMENT}

The authors would like to thank Dr. L. Tremblay, physiotherapist attached to the Parkinson Society of Saguenay, and H. Amamou for assistance. They also wish to thank SOVAR Company for helping the team in their research activities. The authors are grateful to those participants who gave so generously of their time and helped to make this research possible.

\section{REFERENCES}

[1] C. L. Hsu, L. S. Nagamatsu, J. C. Davis, and T. Liu-Ambrose, "Examining the relationship between specific cognitive processes and falls risk in older adults: A systematic review," Osteoporosis Int., vol. 23, pp. 2409-2424, Oct. 2012.

[2] D. Hamacher, N. B. Singh, J. H. Van Dieën, M. O. Heller, and W. R. Taylor, "Kinematic measures for assessing gait stability in elderly individuals: A systematic review," J. R. Soc. Interface, vol. 8, pp. 1682-1698, Dec. 2011.

[3] G. Thrane, R. Joakimsen, and E. Thornquist, "The association between Timed Up and Go test and history of falls: The Tromso study," BMC Geriatrics, vol. 7, pp. 1-7, Jan. 2007.

[4] T. Michikawa, Y. Nishiwaki, T. Takebayashi, and Y. Toyama, "One-leg standing test for elderly populations," J. Orthopaedic Sci., vol. 14, pp. 675-685, 2009.

[5] M. E. Tinetti et al., "A multifactorial intervention to reduce the risk of falling among elderly people living in the community," $N$. Eng. J. Med., vol. 331, pp. 821-827, 1994.

[6] S. W. Muir, K. Berg, B. Chesworth, and M. Speechley, "Use of the Berg Balance Scale for predicting multiple falls in community-dwelling elderly people: A prospective study," Phys. Ther., vol. 88, no. 4, pp. 449-59, Apr. 2008. 
[7] T. Frenken, M. Brell, M. Gövercin, S. Wegel, and A. Hein, "aTUG: Technical apparatus for gait and balance analysis within componentbased Timed Up \& Go using mutual ambient sensors," J. Ambient Intell. Humaniz Comput., vol. 4, pp. 759-778, 2013.

[8] Z. Cris, S. Arash, C.-K. Patricia, G. John, and B. H. Fay, "Assessing mobility at home in people with early Parkinson's disease using an instrumented Timed Up and Go test," Parkinsonism Relat Disord., vol. 17, pp. 277-280, 2011.

[9] S. Sprager and D. Zazula, "Impact of different walking surfaces on gait identification based on higher-order statistics of accelerometer data," in Proc. IEEE Int. Conf. Signal Image Process. Appl., 2011, pp. 360-365.

[10] D. S. Marigold and A. E. Patla, "Adapting locomotion to different surface compliances: Neuromuscular responses and changes in movement dynamics," J. Neurophysiol., vol. 94, pp. 1733-1750, Sep. 2005.

[11] M. Wirz, R. Müller, and C. Bastiaenen, "Falls in persons with spinal cord injury: Validity and reliability of the Berg Balance Scale," Neurorehabil. Neural Repair, 2009.

[12] S. Brassard, M. J.-D. Otis, A. Poirier, and B.-A. J. Menelas, "Towards an automatic version of the Berg balance scale test through a serious game," in Proc. 2nd ACM Workshop Mobile Syst., Appl., Services HealthCare, Toronto, ON, Canada, 2012, pp. 1-6.

[13] J. I. Thomas and J. V. Lane, "A pilot study to explore the predictive validity of 4 measures of falls risk in frail elderly patients," Arch. Phys. Med. Rehabil., vol. 86, pp. 1636-1640, 2005.

[14] R. J. Peterka, "Postural control model interpretation of stabilogram diffusion analysis," Biol. Cybern., vol. 82, pp. 335-343, 2000.

[15] A. L. Hof, M. G. Gazendam, and W. E. Sinke, "The condition for dynamic stability," J. Biomech., vol. 38, pp. 1-8, Jan. 2005.

[16] T. Ersal and K. Sienko, "A mathematical model for incorporating biofeedback into human postural control," J. Neuroeng. Rehabil., vol. 10, no. 14, pp. 1-12, 2013.

[17] A. D. Goodworth, C. Wall, and R. J. Peterka, "Influence of feedback parameters on performance of a vibrotactile balance prosthesis," IEEE Trans. Neural Syst. Rehabil. Eng., vol. 17, no. 4, pp. 397-408, Aug. 2009.

[18] S. J. Wood, F. O. Black, H. G. MacDougall, and S. T. Moore, "Electrotactile feedback of sway position improves postural performance during galvanic vestibular stimulation," Ann. NY Acad. Sci., vol. 1164, pp. 492-8, May 2009.

[19] S. J. M. Bamberg, A. Y. Benbasat, D. M. Scarborough, D. E. Krebs, and J. A. Paradiso, "Gait analysis using a shoe-integrated wireless sensor system," IEEE Trans. Inf. Technol. Biomed., vol. 12, no. 4, pp. 413-423, Jul. 2008.

[20] C. Meng, H. Bufu, and X. Yangsheng, "Intelligent shoes for abnormal gait detection," in Proc. IEEE Int. Conf. Robot. Automat., 2008, pp. 2019-2024.

[21] F. Martinez-Marti et al., "Embedded sensor insole for wireless measurement of gait parameters," Australas Phy. Eng. Sci. Med., vol. 37, pp. 25-35, Mar. 2014.

[22] H. Noshadi et al., "Hermes: Mobile balance and instability assessment system," in Conf. Bio-Inpsired Syst. Signal, Jan. 2010, pp. 264-270.

[23] W. Aner, H. Talia, G. Nir, and M. H. Jeffrey, “Objective assessment of fall risk in Parkinson's disease using a body-fixed sensor worn for 3 days," PLoS ONE, vol. 9, 2014.

[24] A. S. Buchman et al., "Associations between quantitative mobility measures derived from components of conventional mobility testing and Parkinson gait in older adults," PLoS One, vol. 9, no. 1, Jan. 2014.

[25] M. Milosevic, E. Jovanov, and A. Milenkovic, "Quantifying TimedUp-and-Go test: A smartphone implementation," in Proc. IEEE Int. Conf. Body Sensor Netw., 2013, pp. 1-6.

[26] D. Meldrum, A. Glennon, S. Herdman, D. Murray, and R. McConnWalsh, "Virtual reality rehabilitation of balance: Assessment of the usability of the Nintendo Wii ${ }^{\circledR}$ Fit Plus," Disabil. Rehabil., Assist. Technol., vol. 7, pp. 205-210, 2012.

[27] B.-A. J. Menelas and M. J. D. Otis, "A serious game for training balance control over different types of soil," in Serious Games Development and Applications, M. Ma, M. Oliveira, J. Hauge, H. Duin, and K.-D. Thoben, Eds. Berlin, Germany: Springer, 2012, vol. 7528, pp. 31-42.

[28] M. Stalin and C. L. Bennett, "Development of an insole system for real-time capture of ground reaction forces in lower-limb amputees," in Biomed. Eng. Conf., May 2013, pp. 137-138.

[29] A. Cristiani, G. M. Bertolotti, E. Marenzi, and S. Ramat, "An instrumented insole for long term monitoring movement, comfort and ergonomics," IEEE Sensors J., vol. 14, no. 5, pp. 1564-1572, May 2014.
[30] D. H. Gates, J. M. Wilken, S. J. Scott, E. H. Sinitski, and J. B. Dingwell, "Kinematic strategies for walking across a destabilizing rock surface," Gait Posture, vol. 35, pp. 36-42, 2012.

[31] B. A. Springer, R. Marin, T. Cyhan, H. Roberts, and N. Gill, "Normative values for the unipedal stance test with eyes open and closed," $J$. Geriatr. Phys. Ther., vol. 30, no. 1, pp. 8-15, 2007.

[32] F. Menegoni et al., "Mechanisms underlying center of pressure displacements in obese subjects during quiet stance," J. Neuroeng. Rehabil., vol. 8, p. 20, 2011.

[33] A. W. O. Gil et al., "Relationship between force platform and two functional tests for measuring balance in the elderly," Braz. J. Phy. Ther, vol. 15, pp. 429-435, 2011.

[34] M. Piirtola and P. Era, "Force platform measurements as predictors of falls among older people-A review," Gerontology, vol. 52, no. 1, pp. $1-16,2006$.

[35] M. Moghadam et al., "Reliability of center of pressure measures of postural stability in healthy older adults: Effects of postural task difficulty and cognitive load," Gait Posture, vol. 33, pp. 651-655, 2011.

[36] H. van der Kooij, R. Jacobs, B. Koopman, and H. Grootenboer, “A multisensory integration model of human stance control," Biol. Cybern., vol. 80, pp. 299-308, 1999.

[37] A. Mahboobin et al., "Sensory adaptation in human balance control: Lessons for biomimetic robotic bipeds," Neural Netw., vol. 21, pp. 621-627, 2008.

[38] A. Ishida, T. Masuda, H. Inaoka, and Y. Fukuoka, "Stability of the human upright stance depending on the frequency of external disturbances," Med. Biol. Eng. Comput., vol. 46, pp. 213-21, Mar. 2008.

[39] S. Mohapatra, V. Krishnan, and A. S. Aruin, "The effect of decreased visual acuity on control of posture," Clin. Neurophysiol., vol. 123, pp. 173-182, 2012.

[40] S. Mohapatra, V. Krishnan, nd, and A. Aruin, "Postural control in response to an external perturbation: Effect of altered proprioceptive information," Exp. Brain Res., vol. 217, pp. 197-208, 2012.

[41] S. Mohapatra, K. K. Kukkar, and A. S. Aruin, "Support surface related changes in feedforward and feedback control of standing posture," $J$. Electromyogr. Kinesiol., vol. 24, pp. 144-152, 2014.

[42] M. Patel, P. A. Fransson, R. Johansson, and M. Magnusson, "Foam posturography: Standing on foam is not equivalent to standing with decreased rapidly adapting mechanoreceptive sensation," Exp. Brain Res., vol. 208, pp. 519-527, 2011.

[43] B. E. Maki et al., "Reducing fall risk by improving balance control: Development, evaluation and knowledge-translation of new approaches," J. Safety Res., vol. 42, pp. 473-485, 2011.

[44] A. D. Wright, G. A. Heckman, W. E. McIlroy, and A. C. Laing, "Novel safety floors do not influence early compensatory balance reactions in older adults," Gait Posture, vol. 40, pp. 160-165, 2014.

[45] K. B. Cheng, Y.-C. Huang, and S.-Y. Kuo, "Effect of arm swing on single-step balance recovery," Human Movement Sci., vol. 38, pp. 173-184, 2014.

[46] B.-A. Menelas and M. D. Otis, "Toward an automatic system for training balance control over different types of soil," in Virtual, Augmented Reality and Serious Games for Healthcare 1, M. Ma, L. C. Jain, and P. Anderson, Eds. Berlin, Germany: Springer, 2014, vol. 68, pp. 391-408.

[47] J. C. Ayena, L. D. T. Chapwouo, M. J. D. Otis, and B.-A. J. Menelas, "An efficient home-based risk of falling assessment test based on smartphone and instrumented insole," in Proc. IEEE Int. Symp. Med. Meas. Appl., May 2015, pp. 416-421.

[48] D. Gagnon, B. A. J. Menelas, and M. J. D. Otis, "Qualitative risk of falling assessment based on gait abnormalities," in Proc. IEEE Int. Conf. Syst., Man, Cybern., Oct. 2013, pp. 3966-3971.

[49] M. B. Brahem, J. C. Ayena, M. J.-D. Otis, and B.-A. J. Ménélas, "Risk of falling assessment on different types of ground using the instrumented TUG," in Proc. IEEE Int. Conf. Syst., Man, Cybern., Hong Kong, 2015, pp. 1-6.

[50] C. Frank, S. Anthony, K. Lon, H. Jacob, and G. Richard, "A transfer of training study of control loader dynamics," in AIAA Model. Simulat. Technol. Conf., 2011.

[51] M. Gilbert, "Principles of pressure transducers, resonance, damping and frequency response," Anaesthesia Intensive Care Med., vol. 13, pp. 1-6, 2012.

[52] D. T. McRuer and E. S. Krendel, "Mathematical models of human pilot behavior," in NTIS, 1974. 
[53] J. A. Lusardi, C. L. Blanken, M. C. R. Ott, C. A. Malpica, and W. v. Grünhagen, "In flight evaluation of Active inceptor force-feel characteristics and handling qualities," presented at the 68th Annu. Forum Am. Helicopter Soc., Phoenix, AZ, May 2012.

[54] J. S. Bendat and A. G. Piersol, "Data acquisition and processing," in Random Data. New York: Wiley, 2012, pp. 317-357.

[55] M. Cenciarini, P. J. Loughlin, P. J. Sparto, and M. S. Redfern, "Stiffness and damping in postural control increase with age," IEEE Trans. Biomed. Eng., vol. 57, no. 2, pp. 267-275, Feb. 2010.

[56] J. Park, N. Pažin, J. Friedman, V. M. Zatsiorsky, and M. L. Latash, "Mechanical properties of the human hand digits: Age-related differences," Clin. Biomechan., vol. 29, pp. 129-137, Feb. 2014.

[57] A. Eusebio et al., "Resonance in subthalamo-cortical circuits in Parkinson's disease," Brain, vol. 132, pp. 2139-2150, 2009.

[58] R. A. Hess, T. Alsbury, and A. Tencio, "Flight simulator fidelity assessment in a rotorcraft lateral translation maneuver," J. Guidance, Control, Dynamics, vol. 16, pp. 79-85, 1993.

[59] M. J. Campbell, A. J. McComas, and F. Petito, "Physiological changes in ageing muscles," J. Neurol., Neurosurg., Psychiatry, vol. 36, pp. 174-182, 1973.

[60] T. Marasovic, M. Cecic, and V. Zanchi, "Analysis and interpretation of ground reaction forces in normal gait," WSEAS Trans. Syst., vol. 8, pp. 1105-1114, 2009.

[61] M. Nolano et al., "Sensory deficit in Parkinson's disease: Evidence of a cutaneous denervation," Brain, vol. 131, pp. 1903-1911, May 2008.

[62] P. G. Morasso and V. Sanguineti, "Ankle muscle stiffness alone cannot stabilize balance during quiet standing," J. Neurophysiol., vol. 88, pp. 2157-2162, 2002

[63] L. J. Smart, T. A. Stoffregen, and B. G. Bardy, "Visually induced motion sickness predicted by postural instability," Hum. Fact, vol. 44, pp. 451-465, 2002.

[64] R. Stemplewski, J. Maciaszek, W. Osiñski, and R. Szeklicki, “Testretest reliability of measurements of the center of pressure displacement in quiet standing and during maximal voluntary body leaning among healthy elderly men," J. Hum. Kinet., vol. 28, pp. 15-23, Jun. 2011.

[65] A. G. Rodrigo, S. Rony, A. R. Francisco, A. A. David, and A. O. Pablo, "Relationship between unipedal stance test score and center of pressure velocity in elderly," Rev. Esp. Geriatr. Gerontol., vol. 46, no. 5, pp. 256-60, Sep.-Oct. 2011.

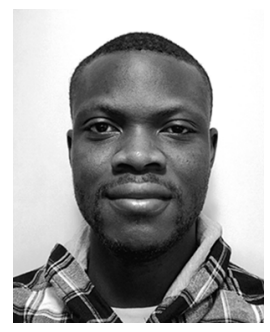

Johannes C. Ayena received the B.S. degree in physics from Faculty of Science Fez, Morocco, in 2009, and the M.Sc. degree in telecommunication engineering from National School of Applied Science, Fez, Morocco, in 2012. He is currently pursuing the $\mathrm{Ph} . \mathrm{D}$. degree in engineering at the Automation and Intelligent 3D Multimodal Interaction Laboratory (LAIMI), Chicoutimi, QC, Canada.

He worked with Water Center Engineering, Fez, Morocco, in 2013. He joined automation and intelligent 3D multimodal interaction laboratory (LAIMI), Chicoutimi, Canada, in 2014. His research activity focuses on bioengineering which include Parkinson's disease, human locomotion and control, and resulting applications in rehabilitation.

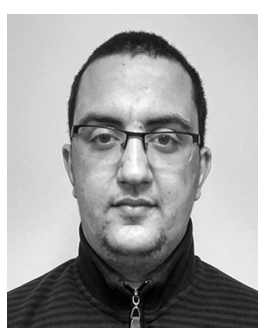

Helmi Zaibi received the B.S. degree in instrumentation and industrial maintenance from the National Institute of Applied Sciences and Technology of Tunis (INSAT), Tunis, Tunisia, in 2013. He is currently pursuing the M.Sc. degree in engineering at the Automation and Intelligent 3D Multimodal Interaction Laboratory (LAIMI), Chicoutimi, QC, Canada.

He worked for the company "MPSE" (Mechatronic Systems Production Engineering), subcontracting of German Dräxlmaier group (automotive wiring: Mercedes, Volkswagen, Audi, BMW), as a supplier quality manager. In 2014, he joined automation and intelligent 3D multimodal interaction laboratory (LAIMI), Chicoutimi, QC, Canada. His research interests include biomechanical modeling, motor control, human locomotion, orthopedic devices and mechanical design.

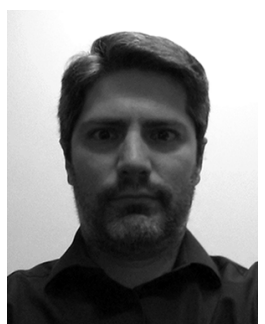

Martin J.-D. Otis received the M.Sc. degree in electrical engineering from University of Sherbrooke, Sherbrooke, QC, Canada, in 2004, and the Ph.D. degree in electrical engineering and robotics from Laval University, Ville de Québec, QC, Canada, in 2009.

$\mathrm{He}$ is an adjunct professor at University of Quebec at Chicoutimi, (2010) and is the LAIMI Director (2013); since 2005 he is a member of REPARTI strategic network (FRQ-NT). His works was performed with the CVSL in the Department of Electrical, Computer Engineering and Robotic Laboratory. He was a Post-Doctoral Fellow at Centre for Intelligent Machines, McGill University, Montréal, QC, Canada, in 2010. His research activity focuses on the design of high-performance haptic interfaces, robot control and interaction.

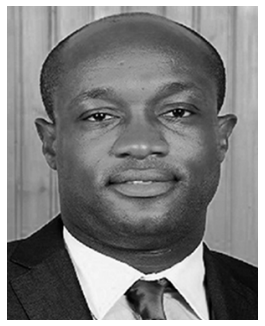

Bob-Antoine J. Ménélas received the M.Sc. degree from University of Angers, Angers, France, in 2006 and the Ph.D. degree in computer science from University of Paris Sud XI, Paris, France, in 2010.

$\mathrm{He}$ has been an Assistant Professor of Computer Science, at the University of Quebec at Chicoutimi (UQAC), Chicoutimi, QC, Canada, since August 2011. Before joining UQAC, he was a postdoctoral fellow at Electrical and Computer Engineering at University of Calgary, Canada. His research interests include haptics, virtual reality, scientific visualization and serious games. 